\title{
Fracture Resistance of Molar Crowns Fabricated with Monolithic All-Ceramic CAD/CAM Materials Cemented on Titanium Abutments: An In Vitro Study
}

Dogan, Derya Ozdemir ; Gorler, Oguzhan ; Mutaf, Burcu ; Ozcan, Mutlu ; Eyuboglu, Gunes Bulut ; Ulgey, Melih

\begin{abstract}
PURPOSE To assess the fracture resistance of single-tooth implant-supported crown restorations made with different CAD/CAM blocks. MATERIALS AND METHODS Thirty-six titanium abutments were put on dental implant analogs (Mis Implant). For each of three test groups ( $\mathrm{n}=12$ /group), implant-supported, cementretained mandibular molar single crowns were produced. Crowns were made of lithium disilicate glass (LD) IPS e.max CAD, feldspathic glass ceramic (FEL) Vita Mark II, and resin nano-ceramic (RNC) Lava Ultimate. The crowns were cemented with self-adhesive resin cement RelyX Unicem 2. After chewing cycling, crowns were tested to failure in a universal testing machine. Fracture values were calculated as initial (F-initial) and maximum fracture (F-max). RESULTS The study groups were ranked, in order of having highest value, (LD $>$ FEL) $>$ RNC for F-initial load value and (LD > RNC) > FEL for F-max load value. This demonstrated that there was no parallel change in the F-initial and F-max values presenting the fracture resistance of specimens. CONCLUSIONS There was no accordance between the F-initial and F-max values of the LD, RNC, and FEL after chewing simulation with thermocycling resembling 5 years of clinical functional use. LD had the highest fracture resistance during the fracture test. RNC had low fracture resistance; however, it had considerably high fracture resistance during the fracture test. FEL had considerably low fracture resistance values.
\end{abstract}

DOI: https://doi.org/10.1111/jopr.12393

Posted at the Zurich Open Repository and Archive, University of Zurich

ZORA URL: https://doi.org/10.5167/uzh-145962

Journal Article

Accepted Version

Originally published at:

Dogan, Derya Ozdemir; Gorler, Oguzhan; Mutaf, Burcu; Ozcan, Mutlu; Eyuboglu, Gunes Bulut; Ulgey, Melih (2017). Fracture Resistance of Molar Crowns Fabricated with Monolithic All-Ceramic CAD/CAM Materials Cemented on Titanium Abutments: An In Vitro Study. Journal of Prosthodontics, 26(4):309-314.

DOI: https://doi.org/10.1111/jopr.12393 


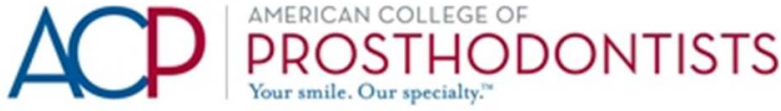

\section{Fatigue behavior of molar crowns fabricated with monolithic all-ceramic CAD/CAM materials cemented on titanium abutments: an in vitro study}

\begin{tabular}{|r|l|}
\hline Journal: & Journal of Prosthodontics \\
\hline Manuscript ID: & JOPR-15-193 \\
\hline Wiley - Manuscript type: & Original Manuscript \\
\hline Index Words: & $\begin{array}{l}\text { dental implant, fatigue resistance, resin nano ceramic, lithium disilicate } \\
\text { ceramic, feldspathic ceramic, CAD/CAM blocks, chewing simulation }\end{array}$ \\
\hline Manuscript Categories: & Academics and Education \\
\hline
\end{tabular}

\section{SCHOLARONE"}

Manuscripts 
Fatigue behavior of molar crowns fabricated with monolithic all-ceramic CAD/CAM materials cemented on titanium abutments: an in vitro study

Derya Ozdemir Dogan ${ }^{1}$, Oguzhan Gorler ${ }^{1}$, Burcu Mutaf ${ }^{2}$, Mutlu Ozcan $^{3}$, Gunes Bulut Eyuboglu ${ }^{4}$, Melih Ulgey $^{1}$

Departments of ${ }^{1}$ Prosthodontics, Cumhuriyet University Faculty of Dentistry, Sivas; ${ }^{2}$ Sultanbeyli Oral Health Center, Istanbul; Turkey, University of Zürich, ${ }^{3} \mathrm{Head}$ of Dental Materials Unit, Center for Dental and Oral Medicine, Clinic for Fixed and Removable Prosthodontics and Dental Materials Science, Zürich, Switzerland; ${ }^{4}$ Department of Restorative Dentistry, Karadeniz Technical University Faculty of Dentistry, Trabzon; Turkey,

Derya Ozdemir Dogan, DDS, PhD, Associate Professor of Department of Prosthodontics, Cumhuriyet University Faculty of Dentistry, Sivas, Turkey; Phone \& Fax: 9034621910 10; E-mail:

drderya@hotmail.com

Oguzhan Gorler, DDS, PhD, Assistant Professor of Department of Prosthodontics, Cumhuriyet University Faculty of Dentistry, Sivas, Turkey; Phone \& Fax: 9034621910 10; E-mail:

drgrı@@ymail.com

Burcu Mutaf, DDS,PhD,, Sultanbeyli Oral Health Center, Istanbul , Turkey; Phone \& Fax: 90216592 41 11; E-mail: mburcu1986@hotmail.com

Mutlu Ozcan, DDS, PhD, Professor of University of Zurich, Head of Dental Materials Unit, Center for Dental and Oral Medicine, Clinic for Fixed and Removable Prosthodontics and Dental Materials

Science, Zürich, Switzerland; Phone \& Fax: 41-44-6345600; E-mail: mutluozcan@hotmail.com

Gunes Bulut Eyuboglu, DDS, Resident of Department of Restorative Dentistry, Karadeniz Technical University Faculty of Dentistry, Trabzon, Turkey; Phone \& Fax: 9034621910 10; E-mail:

gunesbulut@ktu.edu.tr

Melih Ulgey, DDS, Resident of Department of Prosthodontics, Cumhuriyet University Faculty of Dentistry, Sivas, Turkey; Phone \& Fax: 9034621910 10; E-mail: melihulgey@gmail.com

\section{Corresponding Author:}

Dr. Derya Ozdemir Dogan

Department of Prosthodontics

Cumhuriyet University Faculty of Dentistry

Sivas, Turkey

Tel: +90 3462191010

E-mail: dtderya@hotmail.com 
Running title: Fatigue Resistance of all-ceramic crowns

There is no supporting organization.

There is no conflict of interest. 
Abstract

Aims: To assess the fatigue resistance of single-tooth implant-supported crown restorations made with different CAD/CAM blocks.

Methods: Thirty-six titanium abutments were put on dental implant analogs (Mis Implant). For each of three test groups ( $n=12$ per group), implant-supported cement-retained mandibular molar single crowns were produced. Crowns were made of Lithium disilicate glass (LD) IPS e-max CAD, Feldspathik glass ceramic (FEL) Vita Mark II, and Resin nano ceramic (RNC) Lava Ultimate. The crowns were cemented with self-adhesive resin cement RelyX Unicem 2. After chewing cycling, crowns were tested to failure in a universal testing machine. Fracture values were calculated as initial (F-initial) and maximum fracture ( $F-\max )$.

Results: the study groups were ranked, in order of having highest value, (LD > FEL) > RNC for F-initial load value and $(\mathrm{LD}>\mathrm{RNC})>\mathrm{FEL}$ for F-max load value. This demonstrated that there was no parallel change in the F-initial and F-max values.

Conclusions: Within the limitations of the present study, we reached the following conclusions: There was no accordance between the cracking and fracture resistance values of the LD, RNC, and FEL after chewing simulation with thermocycling resembling 5-year of clinical functional use. LD had the highest cracking and fracture resistance during the fracture test. RNC had low cracking resistance, however, it had considerably high fracture resistance during the fracture test. FEL had considerably low cracking and fracture resistance values.

Keywords: dental implant; fatigue resistance; resin nano ceramic material; lithium disilicate ceramic; feldspathic ceramic; CAD/CAM blocks; chewing simulation 


\section{Introduction}

Ceramic-fused-to-metal restorations are accepted as the gold standard in dental implantology. Numerous fabrication techniques have been developed to change their material, shape, and surface characteristics to improve their long-term clinical success and outcome. In implant-supported restorations, titanium is usually preferred because of its well-documented biocompatibility and mechanical properties. Over the last three decades, increased demand for highly esthetic and natural-looking dental restorations has led to the development of new ceramic dental materials and fabrication techniques [1-6]. Manufacturers are constantly introducing newer ceramic materials and improving their existing systems, which have resulted in an increase in all-ceramic restorations and fewer porcelain-to-metal restorations [7].

Improvements in the computer-aided design/computer-aided manufacturing (CAD/CAM) techniques increase the preference of ceramic restorations with recently developed ceramic materials including monolithic or manually veneered all-ceramic systems $[4,5,8]$. Based on the current literature, CAD/CAM-fabricated crowns demonstrate survival rates comparable to conventionally fabricated ones [4]. Based on pertinent literature concerning CAD/CAM used for fabrication of implants and abutments, preliminary proof of concept was established; however, further studies on the performance of these products are required to provide meaningful conclusions to improve their success in vitro and in vivo conditions.

Restorations need to have enough strength to withstand repeated chewing forces under intra-oral conditions. Contemporary or CAD/CAM-fabricated ceramic crowns are subject to fracture during functional use. Crown fractures are one of the most common clinical complications of ceramic crowns. During chewing and trauma, the resulting stresses are transferred to the bone-implant interface, implant, and ceramic component. Fracture potential and degree affect the overall success and outcome of restorative management in long-term use $[6,9,10,11]$. Although research on the materials suitable for CAD/CAM applications are currently the most active field in dental materials [12-15], no studies have evaluated the influence of aging on the strength of CAD/CAM-fabricated molar crowns with currently available ceramic materials. Recent technical development allows the digital manufacturing of monolithic reconstructions with high-performance materials including resin nano ceramic [16].

The ability of molar crowns fabricated with monolithic all-ceramic CAD/CAM materials cemented on titanium abutments to withstand functional forces in the oral cavity is still questionable. Lack of consensus provoked the conduct of this study to compare the fracture resistance of three types of single implant-supported restorations. The aim of this study was to compare the cracking and fracture resistance of three types of implant-supported single crowns fabricated as a representative sample of mandibular molar teeth made of a titanium abutment layered with lithium disilicate, feldspathic glass ceramic, and resin nano ceramic materials after ageing, which that mimics 5 years of clinical service.

\section{Materials and Methods}

The ceramic materials evaluated in this article are listed in Table 1 . Thirty-six titanium abutments (6 $\mathrm{mm}$ in length, $4.8 \mathrm{~mm}$ in width, and $4.8^{\circ}$ in angle of inclination) were placed to simulate a missing mandibular molar on standard dental implant analogs. For each of three test groups ( $n=12$ per group), implant-supported cement-retained mandibular molar single crowns were produced as follows: lithium disilicate glass ceramic (LD group), IPS e-max CAD (Ivoclar Vivadent, Liechtenstein); 
feldspathic glass ceramic (FEL group), Vita Mark II (VITA Zahnfabrik, Bad Säckingen, Germany); and resin nano ceramic (RNC group), Lava Ultimate (3M ESPE, St Paul, MN, USA). All crowns were produced by CAD/CAM) (Figure 1) and their mesiodistal sizes were checked to be $11 \mathrm{~mm}$, buccolingual size, $9 \mathrm{~mm}$, and occlusal fossa thickness, $1.5 \mathrm{~mm}$ (Figure 2). As suggested by the manufacturers, LD crowns were applied to crystallization, RNC crowns were polished, and FEL crowns were glazed. IPS Ceramic Etching Gel containing 5\% hydrofluoric acid (Ivoclar Vivadent, Liechtenstein) was applied to the restoration surface of LD and FEL crowns for 20 seconds as recommended by the manufacturer.

Acid-treated surfaces were washed with distilled water in an ultrasonic bath and dried for about 30 seconds. Then, based on the instructions of manufacturer, Monobond-S (Ivoclar Vivadent, Liechtenstein) was applied to the restoration surface and dried for 60 seconds. RNC crowns and abutments were washed with distilled water for 30 seconds in an ultrasonic bath and all entire surfaces were removed with an air syringe. Then, the cementation process was initiated. After restored crowns were appropriately placed and adapted on abutments with finger pressure, the crowns were cemented with RelyX Unicem 2 self-adhesive resin cement (3M ESPE, St Paul, MN, USA), applying a force of $2 \mathrm{~kg}$; light curing was performed on each surface for 20 seconds. After cementation, all samples were placed in a $37^{\circ} \mathrm{C}$ distilled-water bath for 24 hours.

Testing in the Chewing Simulator (Artificial Mouth)

The specimens ( $\mathrm{n}=12$ for each group) of the study groups were subjected to $45-\mathrm{N}$ load for 1.2 million chewing cycles in the computer-controlled dual-axis chewing simulator (Universal Testing Machine (Zwick ROELL, Ulm, Germany). At the same time, the specimens were exposed to a computercontrolled thermal load using thermocycling $\left(6,000\right.$ cycles, $5^{\circ} \mathrm{C}$ and $55^{\circ} \mathrm{C}$ for 120 seconds each, with a small pause between cold and warm water cycles). This procedure can mimic the performance of all ceramic crowns up to 5 years in the setting of clinical functional use. For recording any events including cracking of the ceramic materials, fractures of the crowns, all specimens were examined daily and after completion of artificial aging in the chewing simulator under a stereomicroscope. Flat, polished antagonists were used according to the loading jig of the static Voss test. The flat antagonists were made of a composite material (Tetric EvoCeram, IvoclarVivadent, Schaan, Liechtenstein) with an elastic modulus similar to that of dentine $[9,17-21]$.

Fracture load measurement

After chewing simulation, the specimens were subjected to fracture load testing in the Universal Testing Machine using a crosshead speed of $0.5 \mathrm{~mm} / \mathrm{min}$ (Zwick/Roell, Ulm, Germany) to measure the fracture load. The load was applied the central fossa of the samples to determine the force required to cracking of the ceramic as F-initial load and fracture of the ceramic totally as F-max load [Figure 4).

\section{Statistical Analysis}

Data were expressed as mean with interquartile range and min-max values. The fracture resistance data were analyzed with ANOVA and post hoc Tukey's test to assess differences in fracture resistance values of the study groups. Analysis was performed by IBM SPSS Statistics software (Mac version 22; IBM Corp, Armonk, NY, USA) A p value of less than 0.05 was accepted as statistical significant. 


\section{Results}

Figure 3 presents the F-initial and F-max load values of fracture test in the LD, RNC, and FEL groups. ANOVA indicated significant differences in mean F-initial and F-max load values among the study groups. Tukey's test revealed that the mean F-initial load value of LD group was significantly increased than those of the RNC and FEL groups (319.9 \pm 112.3 vs. $164.2 \pm 63.7$ and $218.9 \pm 51.8$, respectively; $\mathrm{p}<0.05)$. The F-initial load value of FEL group was higher than that of the RNC group although this difference was not reached statistical significance $(p>0.05)$. Tukey's test presented that the mean F-max load value of LD and RNC group was significantly increased than that of the FEL group ( $2644,5 \pm 546.1$ and $2490.1 \pm 510.9$ vs. $1132.9 \pm 220.4$, respectively; $p<0.05)$. The F-max load value of LD group was higher than that of the RNC group although this difference was not reached statistical significance $(p>0.05)$.

As presented in the Figure 3, the study groups were ranked, in order of having highest value, (LD > $\mathrm{FEL}$ ) > RNC for F-initial load value and ( $L D>$ RNC) $>$ FEL for F-max load value. This demonstrated that there was no parallel change in the F-initial and F-max values.

\section{Discussion}

For the present study, 2 null hypotheses were addressed for the testing of the ultimate failure load: (1) there would be no significant differences in the initial failure resistance values of IPS e-max CAD, Lava Ultimate, and Vita Mark II crown systems, and (2) there would be no significant difference in the maximum failure resistance values of IPS e-max CAD, Lava Ultimate, and Vita Mark II crown systems. The results of this study support the rejection of first and second null hypotheses. To keep the design of all ceramic restoration as the only variable, CAD/CAM technology was used to generate all the crowns identical both in external and internal dimensions and in marginal contours to precisely fit all the titanium abutments in an identical fashion to mimic a missing mandibular molar during the fatigue test in the chewing simulator. This test was adjusted to evaluate 5 -year performance of crowns in the setting of clinical functional use. Fatigue tests seem to have special relevance to several materials including dental ceramics that can be influenced by mechanical fatigue during functional use [22]. In recent years, considerably rapid development has taken place in the area of materials and fabrication techniques used to fabricate more stable and esthetically pleasing ceramic crowns. To present reliable data for objective comparison of in vitro and in vivo performances of newly developed materials requires continuing research with valid test in prosthodontic area.

In the current study, LD provided the highest mean F-initial load value of compared to RNC and FEL. FEL had a F-initial load value more than RNC but with no meaningful difference. LD and RNC offered higher mean F-max load values compared to FEL. Although LD had a F-max load value more compared to RNC, this difference was not meaningful. These results demonstrate that there was no accordance between their F-initial and F-max values. LD provided the highest cracking and fracture resistance values. Although RNC demonstrated low cracking resistance during the measurement of Finitial load, it provided considerably high fracture resistance during the measurement of F-max load. The cracking and fracture resistance of FEL was considerable low compared to other materials except its F-initial load value more compared to RNC.

Recently, different types of ceramic materials such as leucite-reinforced glass ceramic, lithium disilicate glass ceramic, and resin nano ceramic have been used for chair-side fabrication of allceramic restorations using CAD/CAM. The use of monolithic crown restorations has become the preferred treatment option because failure such as cracking is not seen, which does happen with 
zirconia-based ceramic-layered crowns. Each material has its advantages and disadvantages. One of these CAD/CAM ceramics is IPS e.max CAD (Ivoclar VivadentAG, Liechtenstein), an improved glassceramic material with relatively high fracture strength. The IPS e.max CAD is a partially crystallized block of $40 \%$ lithium meta-silicate crystals, which allows the material to be easily milled. After processing the blue block into the desired dental restoration, a recrystallization process takes place at $850^{\circ} \mathrm{C}$ for 10 minutes, through which the lithium meta-silicate is transformed into lithium disilicate crystals. This transformation provides the restoration with its final mechanical and aesthetic properties.[23]

Vitablocks Mark II contains more durable leucite crystals than conventional feldspathic porcelain, and is more aesthetically satisfying. $[24,25]$ The fine crystal microstructure and the CAD/CAM processing technique produce the enamel-like abrasion characteristic of Vita Mark II dental restorations.[26]

Lava Ultimate CAD/CAM Restorative (3M-ESPE TM, St. Paul, USA) is another material used with the CAD/CAM technique. As introduced by its manufacturer, this material is called Resin Nano Ceramic (RNC), which is supposed to be shock-absorbent, resilient, and not brittle, making it ideal for implantsupported crowns. The intra-oral repair of resin-composite crowns can be accomplished by preconditioning, sandblasting, or bur-roughening, followed by the placement of a resin-composite with very similar mechanical and optical properties. The milling time is reduced, the material induces less wear of the milling burs, and more precise margins are possible because the resin content appears to cause less wear on the antagonist cusp (LD and FEL crowns demonstrated more wear than RNC crowns on the antagonist sphere).[27] The clinical potential is enhanced by simple cementation (noHF/silane required), occlusal adjustment, and intra-oral reparability.

In this study, in an effort to standardize and approximate the clinical situation as much as possible, the lower first molar crowns were designed with CAD/CAM system on the standard abutments which have $6 \mathrm{~mm}$ length, $4.8 \mathrm{~mm}$ cervical width and an angle of $4.8^{\circ}$. Occlusal thickness of the restorations was standardized as $1.5 \mathrm{~mm}$, to be able to tolerate occlusal masticatory forces. The restorations were performed in line with their original morphology. They were cemented with self-adhesive resin cement (Rely X Unicem 2 Automix; 3M ESPE). This simplified delivery protocol makes it clinically relevant, fast, appreciated by clinicians, and approved by the block manufacturers.

This study investigated the fatigue resistance of single-tooth implant-supported LD, FEL, and RNC molar restorations. The results showed significant differences among the three groups. The first null hypothesis was partially rejected, that there was no significant difference with respect to fatigue resistance of the three materials used.

LD showed the highest F-initial and F-max values. Although RNC showed the lowest F-max, there was no statistical difference between RNC and LD for F-max. This demonstrated that RNC fractures at higher values because of its resilient structure, even though it is cracked.

Posterior restorations must withstand high masticatory forces. Maximum posterior masticatory forces vary from the 600 to $900 \mathrm{~N}$ depending on facial morphology and age.[28-30] The flexural strength values for FEL were $150 \mathrm{MPa}$, for RNC >204 MPa, and for LD $360 \mathrm{MPa}$ (manufacturers' information). Mean fracture loads for FEL (1141 N), RNC (2490 N), and LD (2644 N) crowns in the present study exceeded physiological chewing force values, which indicates that all three materials tested can be used for cemented implant-supported crown restorations. 
FEL showed the lowest F-max values. The inferior performance of FEL is in agreement with various investigators [31-34] and correlates with lower flexural strength than the two other materials. RNC and LD crowns showed similar resistance despite their different flexural strengths, which was explained by the ratio between strength and elastic modulus (both lower for RNC than for LD). Based on the material's microstructure and physical properties, all-ceramic restorations experience different fatigue behavior in terms of susceptibility to slow cracking growth. The low hardness value of the experimental hybrid-ceramics compared to typical dental ceramics leads to increased surface wear. As a result, the production of large, sliding contact areas results in less stress. Besides that, the wear behavior of the polymer-infiltrated ceramic may be affected by load conditions and the size of the contact area.[35]

Sliding motion of the ceramic indenter causes a frictional force and this creates compressive, tensile, and shear stresses on the ceramic surface. These stresses result in spreading subsurface crackings. Given the composite nature of the experimental hybrid-ceramic materials, it is presumed that the polymer might lubricate the surfaces, which would reduce friction and thus diminish stress- and sliding-induced cracking produced by contact.

Furthermore, the experimental hybrid-ceramic is a damage-resistant material in which the intender produces the surface cracks which pervade the ceramic part, but divert at polymer ceramic interfaces.[36] As no veneering layer is applied in the monolithic CAD/CAM all-ceramic crowns, cyclic loading under wet conditions results in surface cracking formation without any evidence of cracking.[37] This was attributed to the homogenous composition of the CAD/CAM fabricated monolithic ceramics with minimal inherent flaw density and the fully anatomical design of monolithic crowns.

The results of Carvelho et al.'s study comparing the fracture resistance of FEL, RNC, and LD overdenture crown restorations showed that resin nano ceramic and lithium disilicate crowns demonstrated fracture properties more advanced than feldspathic glass ceramic crowns.33 Their study results were consistent with ours for F-max. Another important result of our study was reporting initial values of fracture formation. [38]

Accordingly, crackings were generated in the ceramic body at $231 \mathrm{~N}, 164 \mathrm{~N}$, and $447 \mathrm{~N}$ for FEL, RNC, and LD groups, respectively. The LD structure is the strongest. The RNC is broken due to the high value resilience within the structure even at low values despite the formation of cracks.

Although the outcomes of the current in vitro study cannot be directly compared with in vivo conditions, the fracture strengths in all groups were higher than maximum physiologic masticatory forces. Therefore, it can be assumed that all restorations could potentially withstand physiologic masticatory forces. However, additional research should be performed with the same testing protocol and materials.

According to the limitations of this simulated fatigue resistance study, CAD/CAM crowns placed with a simplified cementation process and made of RNC or LD had significantly higher fatigue resistance than those made of FEL. All the materials survived more than normal range of masticatory forces. The new RNC has the characteristics of major clinical and practical benefits compared with LD (mill time, mill bur usage, polishability, simplicity of insertion, and reparability).

\section{Conclusions}

Within the limitations of the present study, we reached the following conclusions: 
There was no accordance between the cracking and fracture resistance values of the LD, RNC, and FEL after chewing simulation with thermocycling resembling 5 -year of clinical functional use.

LD had the highest cracking and fracture resistance during the fracture test.

RNC had low cracking resistance, however, it had considerably high fracture resistance during the fracture test.

FEL had considerably low cracking and fracture resistance values.

\section{Conflict of interest}

The authors declare that there is no conflict of interest.

\section{References}

1. Sghaireen MG. Fracture Resistance and Mode of Failure of Ceramic versus Titanium Implant Abutments and Single Implant-Supported Restorations. Clin Implant Dent Relat Res. 2013 Oct 9. doi: 10.1111/cid.12160. [Epub ahead of print].

2. Adell R, Lekholm U, Rockler B, Brånemark PI. A 15-year study of osseointegrated implants in the treatment of the edentulous jaw. Int J Oral Surg. 1981 Dec;10(6):387-416.

3. Esposito M, Ardebili Y, Worthington HV. Interventions for replacing missing teeth: different types of dental implants. Cochrane Database Syst Rev. 2014 Jul 22;7:CD003815. doi: 10.1002/14651858.CD003815.pub4.

4. Kapos T, Evans C. CAD/CAM technology for implant abutments, crowns, and superstructures. Int J Oral Maxillofac Implants. 2014;29 Suppl:117-36.

5. Li RW, Chow TW, Matinlinna JP. Ceramic dental biomaterials and CAD/CAM technology: state of the art. J Prosthodont Res. 2014 Oct;58(4):208-16.

6. Ozcan M. Fracture reasons in ceramic-fused-to-metal restorations. J Oral Rehabil. 2003 Mar;30(3):265-9.

7. Santos MJ, Costa MD, Rubo JH, Pegoraro LF, Santos GC Jr. Current all-ceramic systems in dentistry: a review. Compend Contin Educ Dent. 2015 Jan;36(1):38-7.

8. Gulati M, Anand V, Salaria SK, Jain N, Gupta S. Computerized implant-dentistry: Advances toward automation. J Indian Soc Periodontol. 2015 Jan-Feb;19(1):5-10. doi: 10.4103/0972$124 X .145781$.

9. Goodacre CJ, Bernal G, Rungcharassaeng K, Kan JY. Clinical complications in fixed prosthodontics. J Prosthet Dent. 2003 Jul;90(1):31-41.

10. Ralf-Joachim Kohal R, Jolanta Bernadette Kilian JB, Susanne Stampf S, Spies BC. All-ceramic single crown restauration of zirconia oral implants and its influence on fracture resistance: an investigation in the artificial mouth. Materials. 2015 Apr;8:1577-89. doi:10.3390/ma8041577.

11. Kohal RJ, Finke HC, Klaus G. Stability of prototype two-piece zirconia and titanium implants after artificial aging: an in vitro pilot study. Clin Implant Dent Relat Res. 2009 Dec;11(4):3239.

12. Karaokutan I, Sayin G, Kara O. In vitro study of fracture strength of provisional crown materials. J Adv Prosthodont. 2015 Feb;7(1):27-31. 
13. Kurbad A. Provisional polymer-based CAD/CAM implant superstructures. Int J Comput Dent. 2014;17(3):239-51.

14. Corazza PH, Duan Y, Kimpara ET, Griggs JA, Della Bona A. Lifetime comparison of YTZP/porcelain crowns under different loading conditions. J Dent. 2015 Apr;43(4):450-7.

15. Li RW, Chow TW, Matinlinna JP. Ceramic dental biomaterials and CAD/CAM technology: state of the art. J Prosthodont Res. 2014 Oct;58(4):208-16.

16. Joda T, Huber S, Bürki A, Zysset P, Brägger U. Influence of Abutment Design on Stiffness, Strength, and Failure of Implant-Supported Monolithic Resin Nano Ceramic (RNC) Crowns. Clin Implant Dent Relat Res. 2014 Mar 14.

17. Krejci I, Lutz F, Gautschi L. Wear and marginal adaptation of composite resin inlays. J Prosthet Dent. 1994 Sep;72(3):233-44.

18. Mehl C, Scheibner S, Ludwig K, Kern M. Wear of composite resin veneering materials and enamel in a chewing simulator. Dent Mater. 2007 Nov;23(11):1382-9.

19. Sideridou ID, Karabela MM, Vouvoudi ECh. Physical properties of current dental nanohybrid and nanofill light-cured resin composites. Dent Mater. 2011 Jun;27(6):598-607.

20. Spazzin AO, Galafassi D, de Meira-Júnior AD, Braz R, Garbin CA. Influence of post and resin cement on stress distribution of maxillary central incisors restored with direct resin composite. Oper Dent. 2009 Mar-Apr;34(2):223-9.

21. Stawarczyk B, Ender A, Trottmann A, Özcan M, Fischer J, Hämmerle CH. Load-bearing capacity of CAD/CAM milled polymeric three-unit fixed dental prostheses: effect of aging regimens. Clin Oral Investig. 2012 Dec;16(6):1669-77.

22. Silva NR, Bonfante EA, Martins LM, Valverde GB, Thompson VP, Ferencz JL, Coelho PG. Reliability of reduced-thickness and thinly veneered lithium disilicate crowns. J Dent Res. 2012 Mar;91(3):305-10.

23. Christian Ritzberger EA, Wolfram Höland, Arnd Peschke and Volker M. Rheinberger. Properties and Clinical Application of Three Types of Dental Glass-Ceramics and Ceramics for CAD-CAM Technologies. Materials 2010;3(6):3700-3713.

24. Giordano R. Materials for chairside CAD/CAM-produced restorations. J Am Dent Assoc 2006;137 Suppl:14S-21S.

25. Fasbinder DJ. Clinical performance of chairside CAD/CAM restorations. J Am Dent Assoc 2006;137 Suppl:22S-31S.

26. Krejci I, Lutz F, Reimer M. Wear of CAD/CAM ceramic inlays: restorations, opposing cusps, and luting cements. Quintessence Int 1994;25(3):199-207.

27. Tsitrou EA, van Noort R. Minimal preparation designs for single posterior indirect prostheses with the use of the Cerec system. Int J Comput Dent 2008;11(3-4):227-240.

28. Bates JF, Stafford GD, Harrison A. Masticatory function - a review of the literature. III. Masticatory performance and efficiency. J Oral Rehabil 1976;3(1):57-67.

29. Kiliaridis S, Kjellberg H, Wenneberg B, Engstrom C. The relationship between maximal bite force, bite force endurance, and facial morphology during growth. A cross-sectional study. 
Acta Odontol Scand 1993;51(5):323-31.

30. Vult von Steyern P, Jonsson O, Nilner K. Five-year evaluation of posterior all-ceramic threeunit (In-Ceram) FPDs. Int J Prosthodont 2001 Jul-Aug;14(4):379-384.

31. Attia A, Abdelaziz KM, Freitag S, Kern M. Fracture load of composite resin and feldspathic allceramic CAD/CAM crowns. J Prosthet Dent 2006;95(2):117-123.

32. Magne $P$, Knezevic $A$. Simulated fatigue resistance of composite resin versus porcelain CAD/CAM overlay restorations on endodontically treated molars. Quintessence Int 2009;40(2):125-33.

33. Magne $P$, Knezevic A. Influence of overlay restorative materials and load cusps on the fatigue resistance of endodontically treated molars. Quintessence Int 2009;40(9):729-737.

34. Kassem AS, Atta O, El-Mowafy O. Fatigue resistance and microleakage of CAD/CAM ceramic and composite molar crowns. J Prosthodont 2012;21(1):28-32.

35. He LH, Swain M. A novel polymer infiltrated ceramic dental material. Dent Mater 2011;27(6):527-534.

36. Coldea A, Swain MV, Thiel N. Mechanical properties of polymer-infiltrated-ceramic-network materials. Dent Mater 2013;29(4):419-426.

37. Guess PC, Zavanelli RA, Silva NR, Bonfante EA, Coelho PG, Thompson VP. Monolithic CAD/CAM lithium disilicate versus veneered Y-TZP crowns: comparison of failure modes and reliability after fatigue. Int J Prosthodont 2010;23(5):434-442.

38. Carvalho AO, Bruzi G, Giannini M, Magne P. Fatigue resistance of CAD/CAM complete crowns with a simplified cementation process. J Prosthet Dent 2014;111(4):310-317. 
Figure Legends

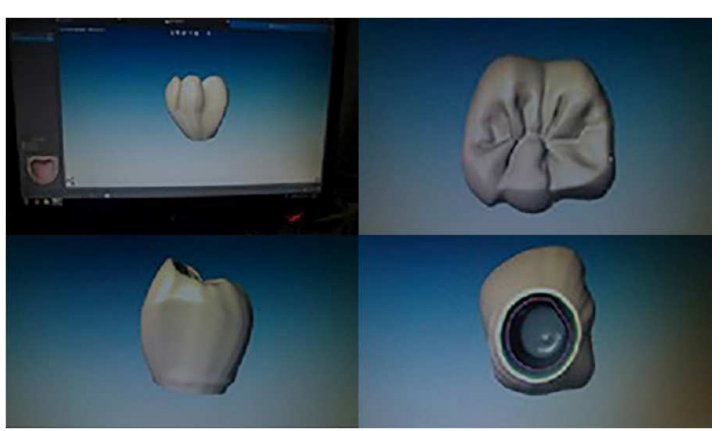

Figure 1. Representative images of the crowns that were produced by CAD/CAM.

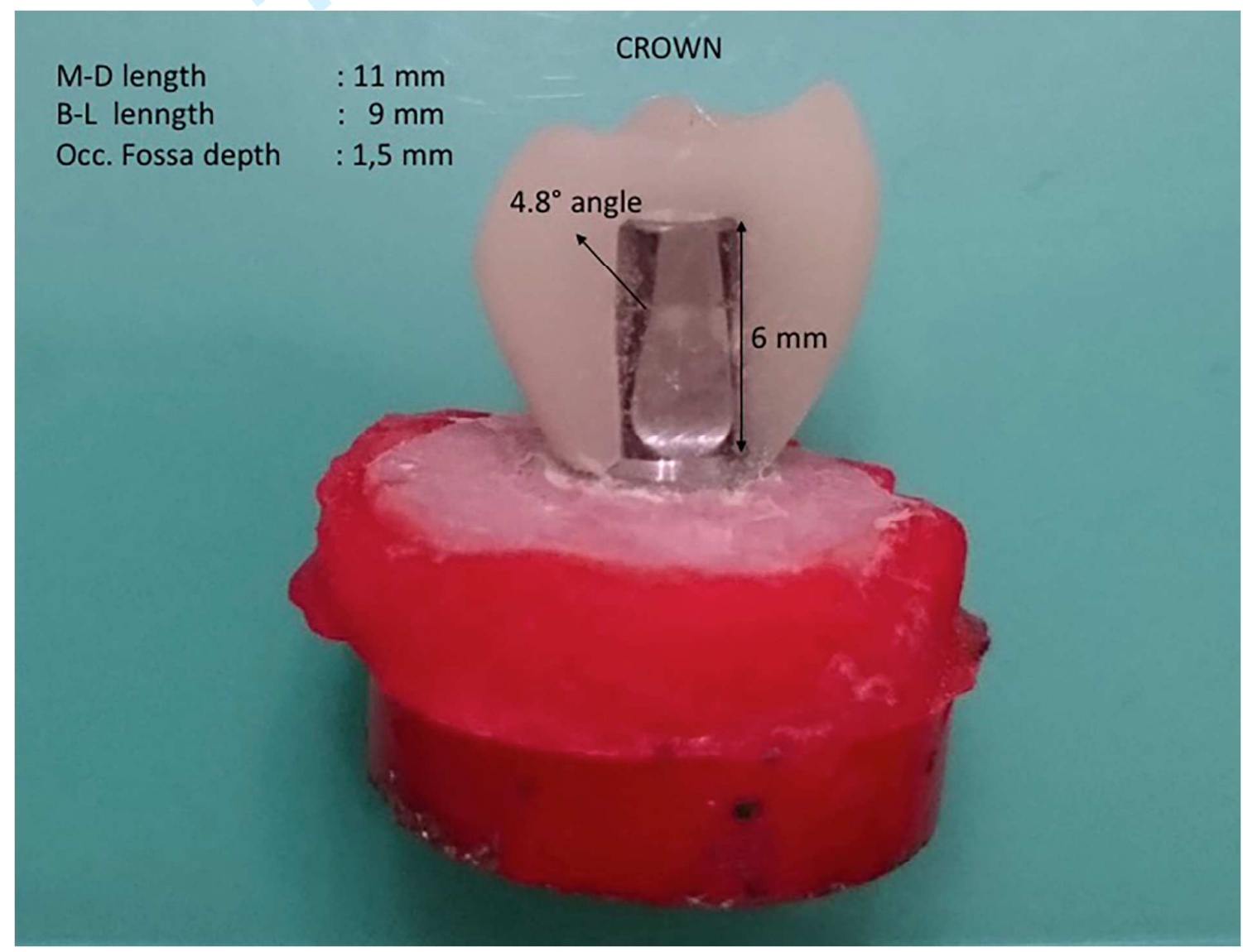

Figure 2. The mesiodistal, buccolingual sizes and occlusal fossa thicknesses of the crowns that were produced by CAD CAM were $11 \mathrm{~mm}, 9 \mathrm{~mm}$ and $1.5 \mathrm{~mm}$, respectively. 


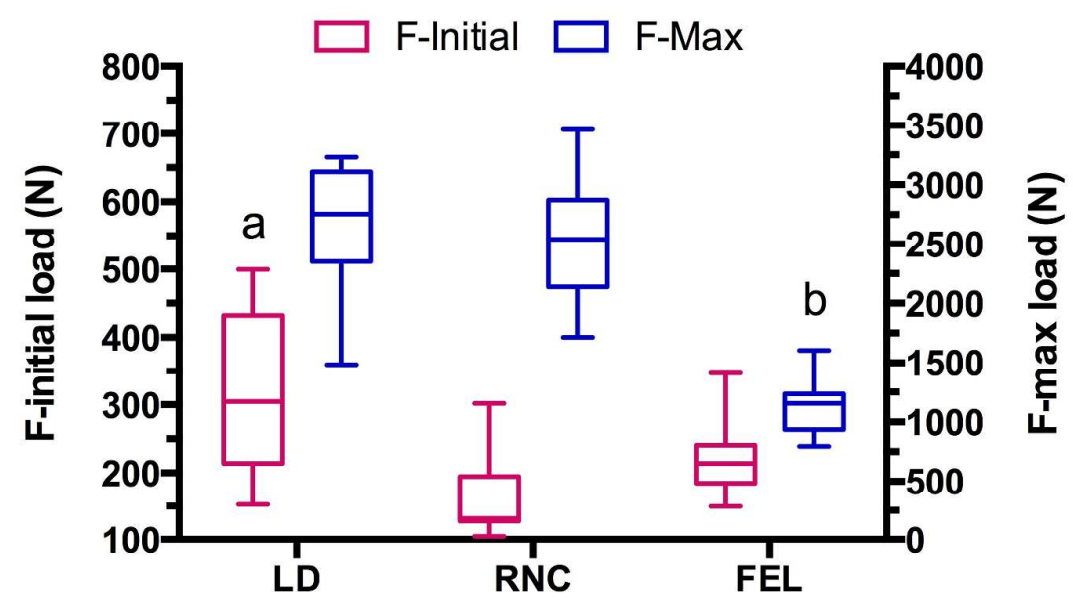

Figure 3. F-initial and F-max load values of LD, RNC, and FEL groups. Data are presented as mean, $25 \%$ and $75 \%$ interquartile ranges, and min and max. LD, IPS e.max CAD; RNC, LAVA Ultimate, and FEL, Vita Mark II. aP<0.05, LD group vs. RNC and FEL groups. bP<0.05, FEL group vs. LD and RNC groups. 


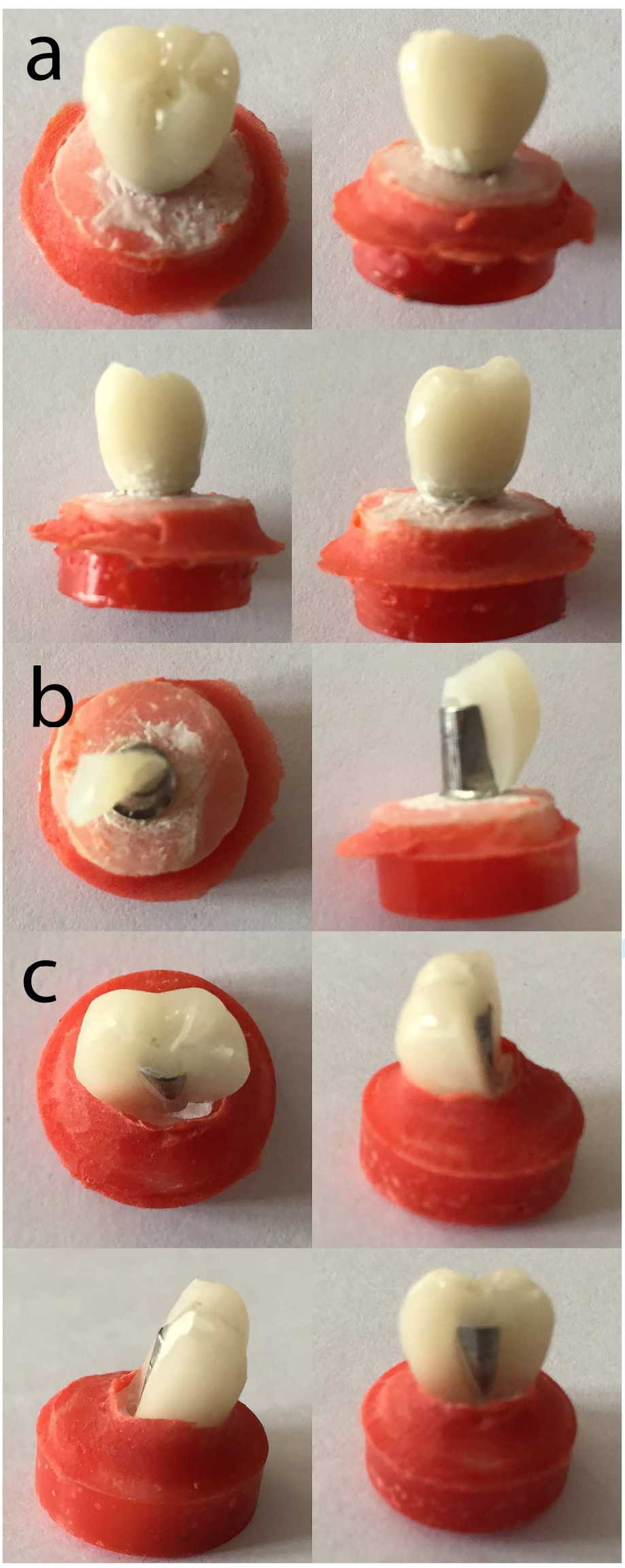

Journal of Prosthodontics 
1

2

3

4

5

6

7

8

9

10

11

12

13

14

15

16

17

18

19

20

21

22

23

24

25

26

27

28

29

30

31

32

33

34

35

36

37

38

39

40

41

42

43

44

45

46

47

48

49

50

51

52

53

54

55

56

57

58

59

60
Figure 4: Before (a) and after $(b, c)$ the examples of images from different angles that showing the diverse types of fracture 
Tables

Table 1. Description of the CAD-CAM ceramic systems used in the study.

\begin{tabular}{|l|l|l|}
\hline CAD/CAM Blocks & Ceramic type & Manifacturer \\
\hline IPS e-max CAD & Lithium disilicate-based glass-ceramic & Ivoclar Vivadent, Liechtenstein \\
\hline Lava Ultimate & Resin-nano ceramic & 3M ESPE, USA \\
\hline Vita Mark II & Feldspathic ceramic & VITA Zahnfabrik, Bad Säckingen \\
\hline
\end{tabular}




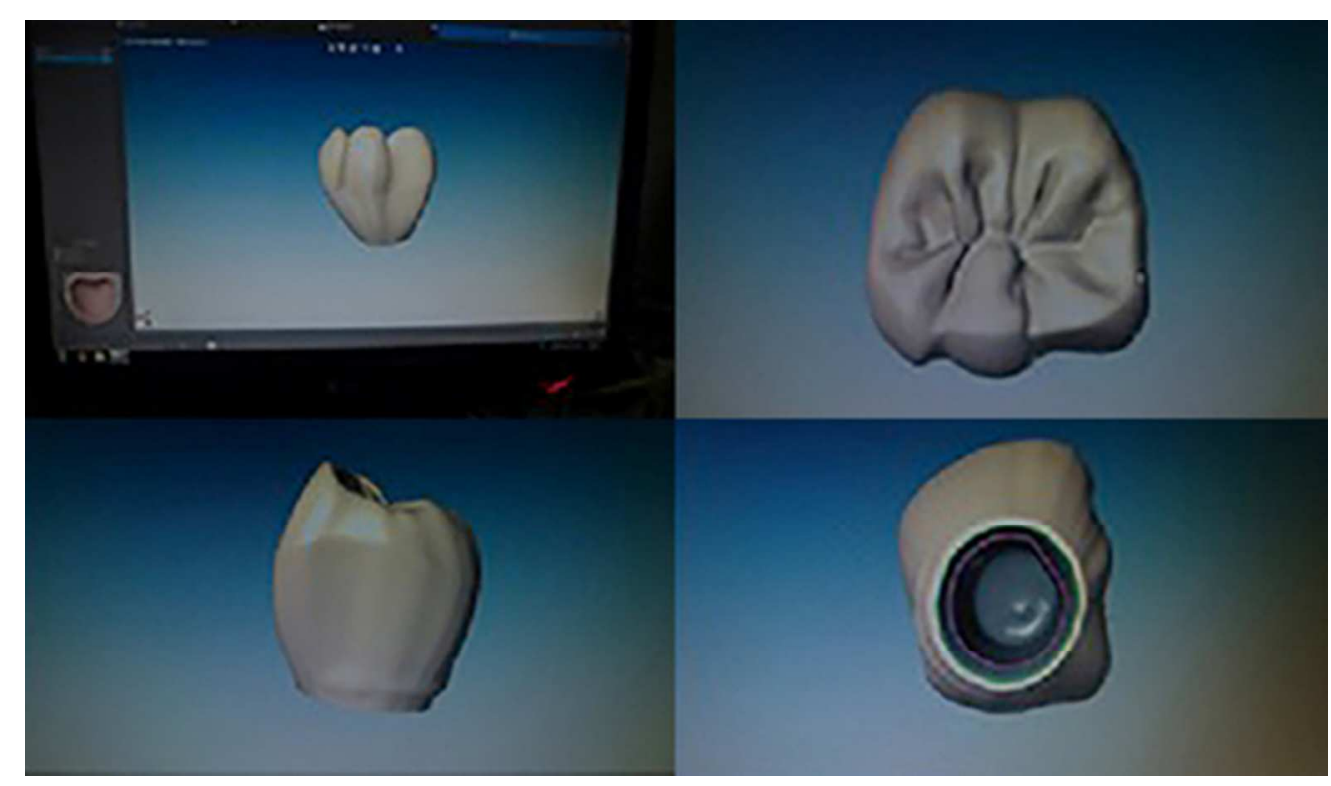

Figure 1. Representative images of the crowns that were produced by CAD/CAM. $44 \times 25 \mathrm{~mm}(300 \times 300 \mathrm{DPI})$ 
Figure 2. The mesiodistal, buccolingual sizes and occlusal fossa thicknesses of the crowns that were produced by CAD CAM were $11 \mathrm{~mm}, 9 \mathrm{~mm}$ and $1.5 \mathrm{~mm}$, respectively.

$58 \times 44 \mathrm{~mm}(300 \times 300$ DPI $)$ 


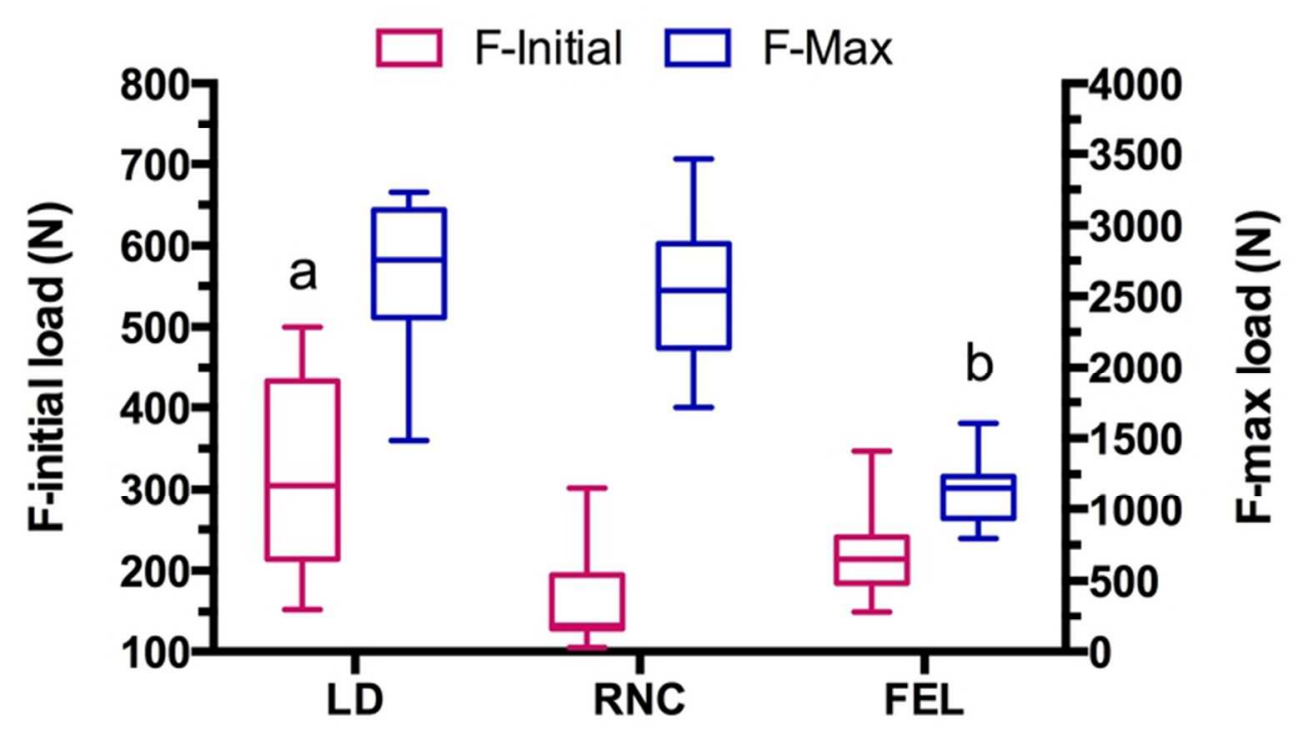

Figure 3. F-initial and F-max load values of LD, RNC, and FEL groups. Data are presented as mean, $25 \%$ and $75 \%$ interquartile ranges, and min and max. LD, IPS e.max CAD; RNC, LAVA Ultimate, and FEL, Vita Mark II. aP $<0.05$, LD group vs. RNC and FEL groups. bP<0.05, FEL group vs. LD and RNC groups. $65 \times 37 \mathrm{~mm}(300 \times 300 \mathrm{DPI})$ 


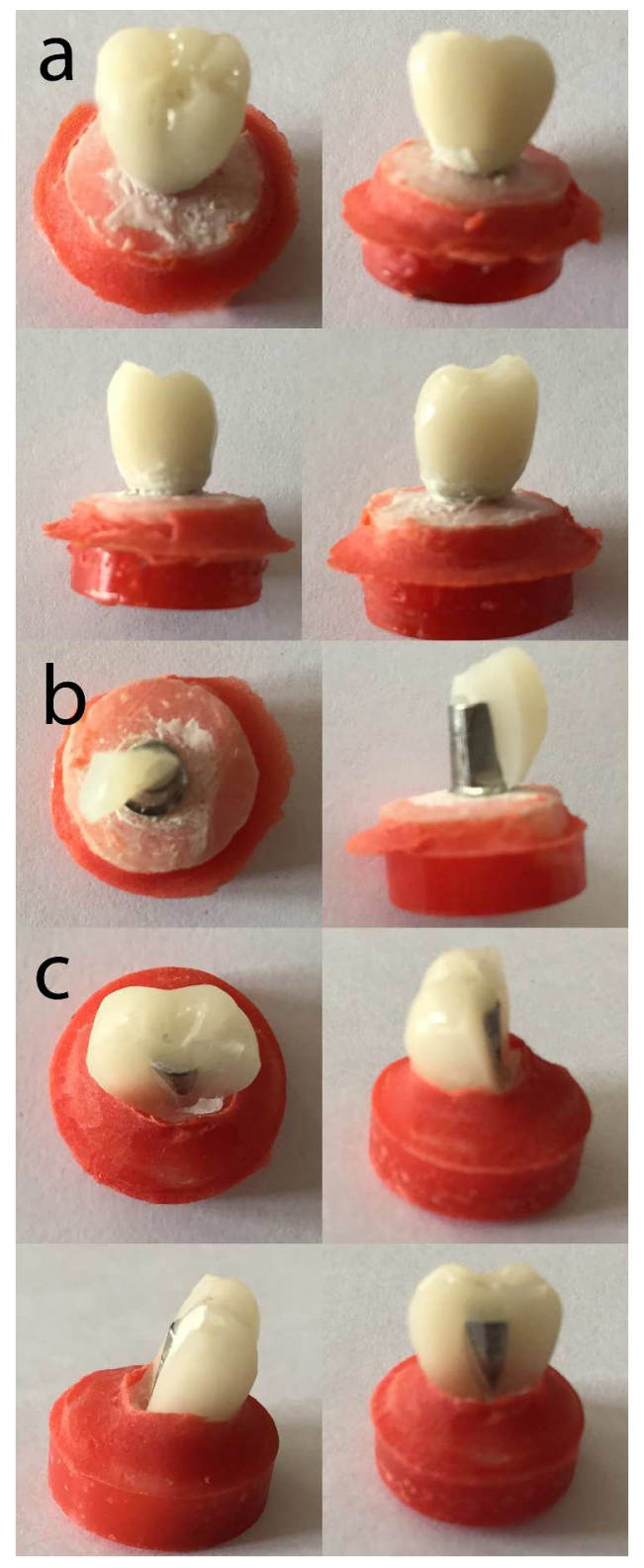

Figure 4: Before $(a)$ and after $(b, c)$ the examples of images from different angles that showing the diverse types of fracture $383 \times 963 \mathrm{~mm}(300 \times 300 \mathrm{DPI})$ 